\title{
An analytical model for predicting rotor broadband noise due to turbulent boundary layer ingestion
}

\author{
Ravish Karve ${ }^{\mathrm{a}, 1}$, David Angland ${ }^{\mathrm{a}, 2, *}$, Thomas Nodé-Langlois $\mathrm{b}^{\mathrm{b}, 3}$ \\ ${ }^{a}$ Faculty of Engineering and the Environment, University of Southampton, Hampshire, \\ SO16 7QF, United Kingdom \\ ${ }^{b}$ Acoustics Methods, Airbus Commercial Aircraft, Toulouse, France
}

\begin{abstract}
A semi-empirical analytical model is developed that predicts the noise produced by a rotor ingesting a boundary layer in proximity to a hard-wall. The rotor boundary layer ingestion noise source is an important source to include when a rotor is installed close to an aircraft fuselage. This is the case for a tail mounted counter rotating open rotor for example. This paper presents three extensions to Amiet's simplified rotor noise model to predict this noise source. The first extension is the method of images, which is used to model the acoustic reflections of the hard-wall. The second extension is an anisotropic velocity spectrum, which is used to model the boundary layer turbulence. The third extension is a numerical switch to account for the partial loading of the rotor as only a part of it is immersed in the boundary layer. The homogeneous anisotropic turbulence model used is a simplification of the actual turbulence the rotor encounters in the boundary layer. In reality, the turbulence in the boundary layer is not homogeneous in the wall-normal direction. Therefore, while the integral length scale in the streamwise direction can be determined from experimental or numerical data, the integral length scale in the wall-normal direction must be chosen empirically. The rotor noise model is validated by comparing its predic-
\end{abstract}

\footnotetext{
* Corresponding author

Email address: d.angland@soton.ac.uk (David Angland)

${ }^{1} \mathrm{PhD}$ Student

${ }^{2}$ Associate Professor

${ }^{3}$ Acoustics Engineer
} 
tions to experimental data at three different advance ratios. The proposed rotor noise model is then used to investigate the effect of the hard-wall on the noise spectrum. The hard-wall affects the downstream directivity more significantly than the upstream directivity for all the rotor operating conditions. Changing the advance ratio of the rotor does not significantly alter the effect that the hard-wall has on the noise spectrum.

Keywords: Open rotor installation noise, Anisotropy, Method of images, Boundary layer ingestion noise

\section{Nomenclature}

$b_{I} \quad$ Amount of the blade immersed in the boundary layer.

C Correlation wavelength.

d Blade span.

$d_{\text {wall }}$ Distance from the rotor hub centre to the wall.

$\mathbf{e}_{\mathbf{i}} \quad$ Unit vector in the $i$ direction.

$\mathcal{F} \quad$ Function used to define anisotropic velocity spectrum.

$f_{x} \quad$ Normalised longitudinal correlation of the $u$ velocity in the $x$ direction.

$f_{y} \quad$ Normalised longitudinal correlation of the $v$ velocity in the $y$ direction.

$F_{i} \quad$ Force on the blade.

$g \quad$ Leading-edge gust response.

$\mathcal{G} \quad$ Function used to define anisotropic velocity spectrum.

$J \quad$ Advance ratio.

$k_{0} \quad$ Acoustic wavenumber.

$k_{i} \quad$ Vortical wavenumber.

$\mathcal{L} \quad$ Acoustic lift.

$L_{11} \quad$ Axial integral length scale.

$L_{22} \quad$ Transverse integral length scale.

$l_{a} \quad$ Axial length scale.

$l_{t} \quad$ Transverse length scale. 
$\mathbf{M}_{\phi} \quad$ Azimuthal Mach number.

$\mathbf{M}_{\mathrm{SF}} \quad$ Mach number of the source with respect to the fluid.

$\mathbf{M}_{\mathrm{SO}}$ Mach number of the source with respect to the observer.

$\mathbf{M}_{\mathbf{x}} \quad$ Mean flow Mach number.

O Location of the observer.

$p \quad$ Acoustic pressure.

$r_{0} \quad$ Distance from the observer to the centre of the engine hub.

$R_{\text {tip }} \quad$ Tip radius of the rotor.

$r_{e} \quad$ Retarded distance to the observer.

$R_{p p} \quad$ Autocorrelation of the pressure.

SO Unit vector from the source to the observer.

S Retarded source location.

s The separation between the two points at which the correlation is considered.

$s_{b} \quad$ Inter-blade spacing.

$S_{p p} \quad$ Total PSD of the acoustic pressure.

$S_{p p}^{I} \quad$ The amplitude term due to the image rotor.

$S_{p p}^{N W} \quad$ PSD of the acoustic pressure without the wall.

$S_{p p}^{P} \quad$ The phase interference term due to the two rotors (real and image) due to the addition of a wall.

$T$ Time between blade-chops as heard by the observer for the real rotor.

$t \quad$ Time.

$T^{\#} \quad$ Time between blade-chops as heard by the observer for the image rotor.

$T^{P 1} \quad$ the time between the $0^{\text {th }}$ blade from the real rotor and the $n^{\text {th }}$ blade from the image rotor chopping an eddy.

$T^{P 2}$ the time between the $n^{\text {th }}$ blade from the real rotor and the $0^{\text {th }}$ blade from the image rotor chopping an eddy.

$T_{1} \quad$ The amount of time between two blades chopping an eddy.

$(u, v, w)$ The turbulent velocities in the $x, y$ and $z$ directions.

$U_{i} \quad$ Mean flow speed. 
$v_{g} \quad$ Gust upwash velocity.

$v_{R} \quad$ Fourier transform of the Gust upwash velocity.

$(\tilde{x}, \tilde{y}, \tilde{z})$ Location of the observer in the blade-fixed frame aligned with the engine.

$(X, Y, Z)$ Location of the observer in the blade-fixed frame aligned with the chord.

$(x, y, z)$ Location of the observer in the engine-fixed frame.

\section{Greek}

$\alpha \quad$ Blade stagger angle.

$\beta \quad$ Compressibility factor.

$\delta \quad$ Height of the boundary layer.

$\Gamma \quad$ Gamma function.

$\gamma \quad$ Azimuthal angle of the blade traversed in the boundary layer.

$\kappa \quad$ Acoustic coupling number.

$\mu_{a} \quad$ Acoustic reduced frequency.

$\mu_{h} \quad$ Hydrodynamic reduced frequency.

$\Omega \quad$ Rotational speed of the blade.

$\omega \quad$ Angular frequency at the source.

$\omega^{\prime} \quad$ Doppler corrected angular frequency at the observer.

$\omega_{I}^{\prime} \quad$ Doppler corrected angular frequency of the image rotor at the observer.

$\phi \quad$ Azimuthal angle of the blade.

$\Phi_{i j} \quad$ PSD of the turbulent velocity fluctuations.

$\psi \quad$ Azimuthal half angle defining the extent of the boundary layer where parts of the blade are immersed.

$\rho_{0} \quad$ Mean density.

$\sigma \quad$ Flow corrected distance to the observer.

$\tau \quad$ Time taken for an acoustic wave to propagate from the blade to the observer.

$\theta \quad$ Axial angle of the observer location.

$\lambda \quad$ Axis of symmetry. 


\section{Acronyms}

BPF Blade Passing Frequency.

CRORS Counter Rotating Open Rotors.

FBN Fan Broadband Noise.

FC3 Fundamental Case 3.

OASPL OverAll Sound Pressure Level.

PSD Power Spectral Density.

PWL sound PoWer Level.

SPL Sound Pressure Level.

URANS Unsteady Reynolds Averaged Navier Stokes.

\section{Introduction}

Counter Rotating Open Rotors (CRORs) are being re-investigated to improve the propulsive efficiency of the next generation of aircraft. While CRORs provide propulsive efficiency advantages, the noise they produce still remains an 5 area of concern [I]. Thus, in order for the CRORs to be adopted for the next generation of aircraft, their noise needs to be reduced significantly [Z] .

Un-installed CROR noise has been studied extensively and the findings from these studies have been adopted to reduce the noise of un-installed open rotors [3]. Installed CROR noise on the other hand, still requires further investigation. This is challenging as open rotors have exposed fans and therefore boundary layers and wakes from the aircraft can interact with the exposed blades. An installation noise source that has been extensively studied, both experimentally and analytically, is the leading-edge noise produced due to the ingestion of a wake generated by an installation pylon [4, 5, 6$]$.

In this paper, the installation noise source considered is the ingestion of a turbulent boundary layer by a rotor. Boundary layer ingestion results in a noise spectrum that shows characteristic haystacks at the Blade Passing Frequencies (BPFs) $[\pi, \mathbb{8}]$. These are caused by the highly anisotropic turbulent structures in the boundary layer. Thus multiple rotor blades can chop the same elongated 
structures in the boundary layer resulting in a highly correlated noise spectra [9]. Additionally, as the rotor is generally installed in proximity to a fuselage, the reflected acoustic waves from the hard-wall could significantly increase the farfield noise [10, II]].

Glegg et al. [10] developed a time-domain rotor noise model that predicts 25 the noise radiated by a rotor ingesting a boundary layer in proximity to a hardwall. The method of Glegg et al. [10] does not model the turbulence in the boundary layer. It instead relies on a four dimensional time and space varying velocity correlation tensor to compute the blade loading by convolving the velocity correlation tensor with a compressible blade response function. This four dimensional velocity correlation tensor must be obtained from an experiment or numerical simulation. While this ensures that the predictions are accurate, it is relatively expensive to obtain the velocity correlation tensor.

The motivation behind this work is to develop a semi-empirical analytical model to predict this noise source by modelling the anisotropic turbulence in the boundary layer. This prediction model can be used in preliminary design studies for example. The rotor noise model is based on Amiet's formulation [I2, [13] for the noise produced by a rotating aerofoil. Amiet's model has been used by several authors to study both the leading and trailing-edge noise of axial fans [14, 15], helicopters [16, 17], and wind turbines [18]. In this paper Amiet's

40 formulation [I2] is extended to account for the features that are required to accurately describe the boundary layer ingestion noise source. There are three modifications that need to be made to Amiet's [1.2] simplified rotor model to enable it to predict the noise generated by a rotor ingesting a boundary layer. The first modification is to account for the presence of the hard-wall. The 45 second is to introduce a turbulence model that accounts for the anisotropy of the turbulence in the boundary layer. The third modification is a numerical switch to account for the partial loading of the rotor since the rotor is only partially immersed in the boundary layer.

The acoustic reflections from the hard-wall are modelled in this paper using the Method Of Images (MOI). The implication of this is that the fuselage is 
modelled by an infinite flat plate. The MOI is a technique in which an image source is added to a real source in a manner that ensures zero normal velocity at the wall [II.]. The MOI has been used to model a wall in the time-domain rotor noise model of Glegg et al. [II0]. Their studies have shown that the wall can significantly affect the sound spectra. The MOI has been also used by Kucukcoskun et al. [20, 2I] to model the hard-wall. They used the MOI with an extended version of Amiet's [1:2] simplified rotor noise model, in which near-field effects and the effects of lean and sweep were added, to model the noise radiated by a fan in the presence of a scattering surface. The solutions compared favourably with results from boundary element simulations and from experimental measurements. This paper introduces the MOI in Amiet's [I2] simplified rotor noise model with blade-to-blade correlation modelled, while assuming that the observer is in the far-field and that the blade has no lean or sweep. However, the assumptions of blade lean and sweep do not detract from is simplified and thus an insight can be obtained into how the hard-wall affects the noise spectrum.

The turbulent structures in the boundary layer are anisotropic [22]. This anisotropy in the turbulent boundary layer is modelled using an anisotropic velocity spectrum. This velocity spectrum is the modified Liepmann spectra of Kerschen and Gliebe [2:3]. This velocity spectrum makes the assumption that the turbulent structures are homogeneous and axisymmetric. Axisymmetric turbulence is defined as turbulence that is symmetric about a specified axis. This implies that the turbulence is cylindrical and can be fully specified by two integral length scales and two turbulence intensities. Thus, an integral length scale in the axial direction and an integral length scale in the transverse direction must be specified along with corresponding turbulence intensities to specify the Power Spectral Density (PSD) of the turbulent velocity fluctuations. It is assumed that the integral length scale in the mean-flow direction is larger than in the other directions. This assumption is a good approximation of the turbulence in a boundary layer as the turbulent structures in the log-layer are known to 
be long cylindrical structures [22, [24, [25]. While boundary layer turbulence is axisymmetric, it is not homogeneous in the wall-normal direction. The rotor noise model presented takes into account the varying turbulence parameters with varying wall-normal locations, and thus partially accounts for the inhomogeneity of the flow. However, the homogeneous axisymmetric turbulence model used in this paper is an approximation of the real turbulence in the boundary layer and a degree of empiricism is introduced into the rotor noise model in order to approximate this turbulence. Additionally, the boundary layer is assumed to be minimally distorted due to the presence of the rotor. A rotor operating at a high thrust could significantly distort the boundary layer. This effect is not accounted for in this model. The anisotropic turbulence spectrum of Kerschen and Gliebe [23] has been used in cascade models by several authors [266, [27].

This paper is organised as follows. Section [2] presents Amiet's formulation for a translating aerofoil and its extension to account for a hard-wall using the MOI. Section [2.4 outlines the extension to Amiet's simplified rotor noise model including a hard-wall and with blade-to-blade correlation modelled. Section 2.5 details the anisotropic turbulence velocity spectrum that is used to model the boundary layer turbulence. Section [2.6 presents a switch to account for the partial loading of the rotor. After the three modifications to Amiet's simplified rotor noise model have been detailed, Section $\$$ presents the results. First, the proposed rotor noise model is validated by comparing with experimental measurements. Next, the effect of the hard-wall on the radiated noise is examined.

\section{Rotor noise model}

In this section, the formulation for the acoustic PSD of a rotor ingesting isotropic turbulence is presented. The formulation procedure proposed by Amiet [28] begins with the computation of the PSD for a translating aerofoil. This PSD is then corrected using a Doppler factor and the result is then averaged over all azimuthal angles. This procedure neglects acceleration effects. This assumption is accurate if the rotational frequency of the rotor is much larger 
than the frequency of the turbulent structures impinging on the rotors. This lower frequency bound is approximately the shaft frequency of the rotor $(\Omega)$ [2Q9]. As there will be correlation peaks at the Blade Passing Frequencies (BPFs) $B \Omega$, where $B$ is the number of blades, this model is applicable for the frequency range of interest for the case of a fan ingesting a turbulent boundary layer.

\subsection{Coordinate systems}

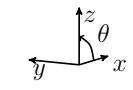

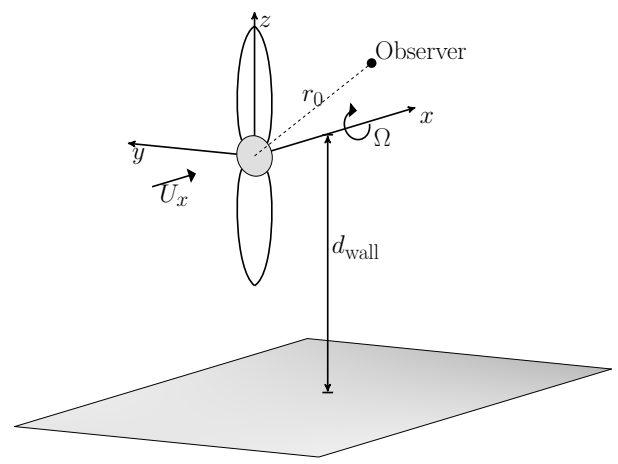

(a)

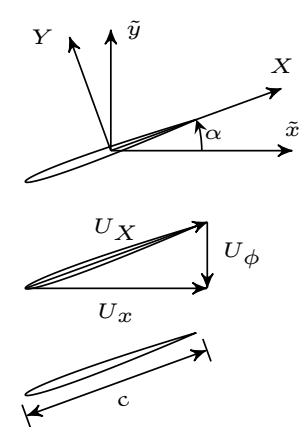

(b)

Figure 1: The coordinate systems used in the rotor noise model. (a)The engine-fixed coordinate system; (b)The two on-blade coordinate systems.

The coordinate systems used in the derivation of the rotor noise model are shown in Fig. $\mathbb{\square}$. The far-field coordinate system is fixed to the engine hub. The mean-flow is in the positive $x$ direction. The observer is located at a distance $r_{0}$ from the engine hub, at an angle $\theta$ to the positive $x$ direction, and is located in the $x-z$ plane. The coordinates of the observer from the real and image sources in the engine-fixed frame are then given by,

$$
\begin{aligned}
\mathbf{O} & =\left(r_{0} \cos \theta, 0, r_{0} \sin \theta\right), \\
\mathbf{O}^{\#} & =\left(r_{0} \cos \theta, 0,-r_{0} \sin \theta-2 d_{\text {wall }}\right),
\end{aligned}
$$

where quantities with a superscript \# indicate values measured from the mirror source. All the other quantities that will be derived can be obtained for the 
mirror rotor by substituting $\mathbf{O}$ with $\mathbf{O}^{\#}$ where necessary and additionally accounting for the opposite rotation of the mirror rotor. The source velocity with respect to the observer and the fluid can be defined as,

$$
\begin{aligned}
& \mathbf{M}_{\mathrm{SO}}=\left(0,-M_{\phi} \cos \phi,-M_{\phi} \sin \phi\right), \\
& \mathbf{M}_{\mathrm{SF}}=\left(-M_{x},-M_{\phi} \cos \phi,-M_{\phi} \sin \phi\right),
\end{aligned}
$$

where $\phi$ is the azimuthal angle of the blade defined in Fig. B, $\mathbf{M}_{\mathrm{SO}}$ is the Mach number of the source relative to the observer, $\mathbf{M}_{\mathrm{SF}}$ is the Mach number of the source relative to the fluid, $M_{x}=U_{x} / c_{o}$, and $M_{\phi}=U_{\phi} / c_{0}$ where $U_{\phi}$ is the azimuthal velocity of the blade $R \Omega$. The retarded source position at emission time $T_{e}$ can be determined using the relationship $c_{0} T_{e}=r_{e}$,

$$
\mathbf{S}=\left(r_{e} M_{x}, 0,0\right) .
$$

The retarded distance to the observer is then given by,

$$
r_{e}=\frac{r_{0}\left(\sqrt{1-M_{x}^{2} \sin ^{2} \theta}-M_{x} \cos \theta\right)}{\beta^{2}},
$$

where $\beta=\sqrt{1-M_{X}^{2}}$ is the compressibility factor and the retarded observer vector is found as $\mathbf{O}^{\prime}=\mathbf{O}-\mathbf{S}-\mathbf{M}_{\mathbf{S F}} r_{e}$,

$$
\mathbf{O}^{\prime}=\left(r_{0} \cos \theta, r_{e} M_{\phi} \cos \phi, r_{0} \sin \theta+r_{e} M_{\phi} \sin \phi\right) .
$$

Using Eqs. (四) and (四), the unit vector in the direction from the retarded source to the observer can be defined as,

$$
\widehat{\mathbf{S O}}=\frac{\left(x-r_{e} M_{x}, 0, z\right)}{r_{e}},
$$

where the observer is located at $(x, 0, z)$. Fig. $\square$ shows a section of unrolled blades at a particular radius from the engine hub. Three coordinate systems have been used, one engine-fixed $(x, y, z)$ and two blade-fixed coordinate systems, $(\tilde{x}, \tilde{y}, \tilde{z})$ and $(X, Y, Z)$. For a blade at an azimuthal angle $\phi=\Omega t$ and for a blade segment with stagger angle $\alpha$ the coordinate systems are related by,

$$
\left(\begin{array}{l}
\tilde{x} \\
\tilde{y} \\
\tilde{z}
\end{array}\right)=\left[\begin{array}{ccc}
1 & 0 & 0 \\
0 & \cos \phi & \sin \phi \\
0 & -\sin \phi & \cos \phi
\end{array}\right]\left(\begin{array}{l}
x^{\prime} \\
y^{\prime} \\
z^{\prime}
\end{array}\right),
$$




$$
\left(\begin{array}{l}
X \\
Y \\
Z
\end{array}\right)=\left[\begin{array}{ccc}
\cos \alpha & \sin \alpha & 0 \\
-\sin \alpha & \cos \alpha & 0 \\
0 & 0 & 1
\end{array}\right]\left(\begin{array}{l}
\tilde{x} \\
\tilde{y} \\
\tilde{z}
\end{array}\right) .
$$

2.2. Formulation for the noise produced by a translating aerofoil in proximity to a hard-wall

In this section Amiet's formulation [28] for the noise produced by a translating aerofoil ingesting turbulence is extended using the MOI to include the effect of a hard-wall. This is the first step in deriving the noise produced by a rotating aerofoil as this result will be azimuthally averaged and frequency corrected to account for the rotation of the aerofoil.

A single frequency component of a turbulent gust interacting with the leadingedge of the aerofoil is given by,

$$
\tilde{v}_{g}\left(k_{X}, k_{Y}, k_{Z}\right)=v_{R}\left(k_{X}, k_{Y}, k_{Z}\right) e^{-\mathrm{i}\left[k_{X}\left(X_{C}-U_{X} t\right)+k_{Y} Y+k_{Z} Z\right]},
$$

where $v_{g}$ is the gust upwash velocity, $v_{R}$ is the spatial Fourier transform of the gust velocity, $\mathbf{k}=\left(k_{X}, k_{Y}, k_{Z}\right)$ defines the wavenumber vector, and $U_{X}$ is the chord-wise component of the flow. The pressure jump on the flat-plate due to the interaction with this gust is then given by,

$\Delta p\left(X, Y, Z, t, k_{X}, k_{Z}\right)=2 \pi \rho_{0} U_{X} v_{R}\left(k_{X}, k_{Z}\right) g^{L E}\left(X, k_{X}, k_{Z}, M_{X}\right) e^{\mathrm{i}\left(k_{X} U_{X} t-k_{Y} Y-k_{Z} Z\right)}$,

where $g^{L E}$ is the non-dimensional gust response function for leading-edge interactions as defined by Amiet [표]. The loading of the blade due to the interaction with this gust can be modelled as a point dipole. For a dipole, located at the coordinates $\left(X_{C}, Z_{C}\right)$ and aligned with the $Y$-axis, the loading is given by [12],

$$
\begin{aligned}
p^{(n)}(X, \mathcal{C}, Z, \omega)= & \frac{\mathrm{i} k_{o} \rho_{o} Y}{2 \sigma^{2}} e^{\mathrm{i}\left[\omega t+\mu\left(M_{X} X-\sigma\right)\right]} \int_{-d}^{d} \int_{-b}^{b} e^{-\mathrm{i} \mu\left(M_{X} X_{C}-\frac{X X_{C}+\beta^{2} Z z_{C}}{\sigma}\right)} \times \\
& \iint_{-\infty}^{\infty} v_{R}\left(K_{X}, k_{Y}, k_{Z}\right) g^{L E}\left(X, K_{X}, k_{Z}, M_{X}\right) e^{-\mathrm{i}\left[k_{Y}(n \mathcal{C})+k_{Z} Z\right]} \mathrm{d} k_{Y} \mathrm{~d} k_{Z} \mathrm{~d} X_{C} \mathrm{~d} Z_{C},
\end{aligned}
$$


where $k_{0}=\omega / c_{0}$ is the acoustic wave number, $\mu=M_{X} k_{X} / \beta^{2}$ is the acoustic reduced frequency, $\sigma=\sqrt{X^{2}+\beta^{2}\left(Y^{2}+Z^{2}\right)}$ is the flow corrected distance to the observer, $K_{X}=\omega / U_{X}$, and $d$ and $b$ represent the half span and half chord of the rotor blade respectively. Additionally in Eq. (ए2), the distance $n \mathcal{C}$ has been introduced to represent the normal distance between the $0^{\text {th }}$ and $n^{\text {th }}$ blades. Therefore, the far-field pressure can be obtained as,

$p^{(n)}(X, \mathcal{C}, Z, \omega)=\frac{\mathrm{i} \pi k_{o} \rho_{o} Y b}{2 \sigma^{2}} e^{\mathrm{i} \mu\left(M_{X} X-\sigma\right)} \mathcal{L}\left(k_{X}, K_{Z}, \kappa\right) \int_{-\infty}^{\infty} v_{R}\left(K_{X}, k_{Y}, K_{Z}\right) e^{-\mathrm{i} k_{Y} n \mathcal{C}} \mathrm{d} k_{Y}$,

where $\kappa=\left(k_{0}\left(M_{X}-X / \sigma\right)\right) / \beta^{2}$ is the acoustic coupling wavenumber, $K_{Z}=$ $\mu \beta^{2} Z / \sigma$, and the acoustic lift $\mathcal{L}$ is defined as,

$$
\mathcal{L}\left(k_{X}, K_{Z}, \kappa\right)=\frac{1}{b} \int_{-b}^{b} g^{L E}\left(X_{C}, k_{X}, K_{Z}, M_{X}\right) e^{-\mathrm{i} \kappa X_{C}} \mathrm{~d} X_{C}, .
$$

The detailed formulation of the acoustic lift can be found in [ $[28]$.

The noise spectrum with blade-to-blade correlation is determined by computing the cross-correlation of the $0^{\text {th }}$ and $n^{\text {th }}$ blade,

$$
S_{p p}^{(n)}(X, \mathcal{C}, Z, \phi, \omega)=\frac{\pi U_{X}}{R} \mathbb{E}\left[p^{(0)}\left(r_{0}, \theta, \phi, \omega\right)\left(p^{(n)}\left(r_{0}, \theta, \phi, \omega\right)\right)^{*}\right] .
$$

The effect of the hard-wall is now included in Eq. (四) by adding a mirror rotor at a distance of $2 d_{\text {wall }}$ from the real rotor hub. This gives rise to four terms,

$$
S_{p p}^{(n)}\left(r_{0}, d_{\mathrm{wall}}, \theta, \phi, \omega\right)=S_{p p}^{N W}+S_{p p}^{P 1}+S_{p p}^{P 2}+S_{p p}^{I},
$$

which are defined as,

$$
\begin{aligned}
S_{p p}^{N W}(X, \mathcal{C}, Z, \phi, \omega) & =\pi U_{X} d\left(\frac{k_{o} \rho_{o} Y b}{\sigma^{2}}\right)^{2}\left|\mathcal{L}\left(k_{X}, K_{Z}, \kappa\right)\right|^{2} \tilde{\Phi}_{v v}\left(K_{X}, k_{Y}, K_{Z}\right), \\
S_{p p}^{P 1}\left(X, \mathcal{C}, Z, d_{\text {wall }}, \phi, \omega\right) & =[\mathrm{A}] \tilde{\Phi}_{v v}\left(K_{X}, k_{Y}, K_{Z}\right)\left|\mathcal{L}\left(\mathcal{L}^{\#}\right)\right|, \\
S_{p p}^{P 2}\left(X, \mathcal{C}, Z, d_{\text {wall }}, \phi, \omega\right) & =[\mathrm{A}]^{*} \tilde{\Phi}_{v v}\left(K_{X}, k_{Y}, K_{Z}\right)\left|\mathcal{L}^{*} \mathcal{L}^{\#}\right|, \\
S_{p p}^{I}\left(X, \mathcal{C}, Z, d_{\text {wall }}, \phi, \omega\right) & =\pi U_{X} d\left(\frac{k_{o} \rho_{o} Y^{\#} b}{\sigma^{2}}\right)^{2}\left|\mathcal{L}^{\#}\left(k_{X}, K_{Z}, \kappa\right)\right|^{2} \tilde{\Phi}_{v v}\left(K_{X}, k_{Y}, K_{Z}\right),
\end{aligned}
$$


where quantities with a superscript $\#$ indicate values at the mirror source and the amplitude term $\mathrm{A}$ is given by,

$$
\mathrm{A}=\pi U_{X} d\left(\frac{k_{o} \rho_{o} b Y Y^{\#}}{\sigma \sigma^{\#}}\right)^{2} e^{\mathrm{i} \mu\left(M_{X}\left(X-X^{\#}\right)+\left(\sigma^{\#}-\sigma\right)\right)},
$$

where,

$$
\tilde{\Phi}_{v v}\left(K_{X}, k_{Y}, K_{Z}\right)=\int_{-\infty}^{+\infty} \Phi_{v v}\left(K_{X}, k_{Y}, K_{Z}\right) e^{-\mathrm{i} k_{Y} n \mathcal{C}} \mathrm{d} k_{Y}
$$

and the transverse velocity spectrum $\Phi_{v v}\left(k_{x}, k_{y}, k_{z}\right)$ is defined as,

$$
\Phi_{v v}\left(k_{x}, k_{y}, k_{z}\right)=\frac{\pi^{2}}{R d} \mathbb{E}\left[v_{R}\left(k_{x}, k_{y}, k_{z}\right) v_{R}^{*}\left(k_{x}, k_{y}, k_{z}\right)\right] .
$$

125

Eq. ([6) and Eq. ([ד) represent the cross PSD of the acoustic pressure of two blades separated by a distance $n \mathcal{C}$ normal to the blade. This differs from Amiet's [28] original formulation, which gives the PSD of the acoustic pressure of a single blade. The cross PSD contains both real and imaginary parts. Amiet's original formulation can be obtained from Eq. (마) and Eq. (파) by setting the blade normal distance $n \mathcal{C}$ to 0 .

$S_{p p}^{N W}$ (no-wall) represents Amiet's original formulation for the noise radiated by a flat plate ingesting turbulence in the absence of the hard-wall. Eq. (ए6) and Eq. (ㅁ) show that the hard-wall has added three additional terms to Amiet's original formulation [ $[28]$, where each term is the cross PSD of the acoustic pressure between two blades. The additional terms are divided into two phase terms (with a superscript $P$ ), due to the interference between the rotor and image rotor, and one amplitude term due to the image rotor (with a superscript $I)$. The phased interference terms $S_{p p}^{P 1}$ and $S_{p p}^{P 2}$ represent the interference of 140 the rotor and image rotor sources. The interference of the acoustic waves in these terms is due to the factor $e^{ \pm \mathrm{i} \mu\left(M_{X}\left(X-X^{\#}\right)+\left(\sigma^{\#}-\sigma\right)\right)}$. This factor can be imaginary and thus changes the phase of the solution. This term is a function of the distance to the observer from the real and image sources and thus represents the phase difference in the acoustic waves that arrive at the observer from the 

or destructive interference.

The amplitude term $S_{p p}^{I}$ gives the amplitude contribution due to the image rotor. In the absence of the phase interference terms $\left(S_{p p}^{P 1}\right.$ and $\left.S_{p p}^{P 2}\right)$, it would represent the doubling of acoustic pressure.

Eq. (एᄄ]) and Eq. (피 represent the extension to account for the hardwall. This extension is important to model the boundary layer ingestion noise source. If the hard-wall was not accounted for there would be a discrepancy in the amplitude of the predicted noise spectrum. While the above formulation considers the cross-correlation between two aerofoils separated by a distance $n \mathcal{C}$, the next step is to include the effects of blade-to-blade correlation by considering the time between eddy chops as heard by the observer.

\subsection{Formulation for the noise produced by a translating aerofoil in proximity} to a hard-wall with blade-to-blade correlation

In this section the kinematics of two rotor blades chopping the same eddy are examined and the time between two blades chopping a single eddy is determined. This is done by considering the cross-correlation of two blades chopping the same eddy. To account for multiple blade passes the cross-correlation function of the acoustic pressure of the $0^{\text {th }}$ blade and the $n^{\text {th }}$ blade can be defined following a similar method to Amiet [12] as,

$$
R_{p p}(x, y, \tau)=\sum_{n=-\infty}^{+\infty} R_{p p}^{(n)}(x, y, \tau-n T),
$$

where $n T$ is the time between eddy chops as heard by the observer. Each of the four terms in Eq. ए6 will have different times between blade chops as heard by the observer. For the first and fourth terms of Eq. ए6, $T$ represents the time between the $0^{\text {th }}$ and $n^{\text {th }}$ blade of the real and image rotor chopping an eddy. For the phase interference terms, $T$ represents the time between the $0^{\text {th }}$ blade of the real rotor chopping an eddy and the $n^{\text {th }}$ blade of the image rotor (or vice-versa) chopping the same eddy. The next step is to determine these time differences. 


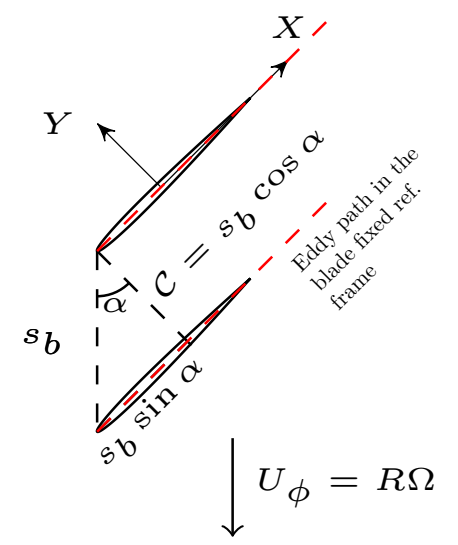

Figure 2: Schematic showing the correlation distance between two sucessive blades chopping the same eddy.

Fig. 『 shows a schematic of two consecutive blades in a blade row. The blades are separated by the inter-blade spacing $s_{b}=U_{\phi} T_{0}$ where $T_{0}=2 \pi / B \Omega$. In the reference frame fixed to a rotor blade, the eddy is moving parallel to the chord at a speed $\sqrt{U_{x}^{2}+U_{\phi}^{2}}$. The time between eddy chops $\left(T_{1}\right)$ by two consecutive blades is then given by,

$$
T_{1}=\frac{s_{b} \sin \alpha}{\sqrt{U_{x}^{2}+U_{\phi}^{2}}}=\frac{U_{\phi}^{2} T_{0}}{U_{x}^{2}+U_{\phi}^{2}},
$$

where the fact that the blade is unloaded (see Fig. $\mathbb{U}$ ) is used to determine $\sin \alpha$. In Eq. ([प]) a blade-normal wavenumber $K_{Y}$ has been introduced to model blade-to-blade correlation. The effective wavelength for this wavenumber is then the perpendicular distance between the consecutive blades given by,

$$
\mathcal{C}=s_{b} \cos \alpha=\frac{U_{x} U_{\phi} T_{0}}{\sqrt{U_{x}^{2}+U_{\phi}^{2}}} .
$$

The time between the eddy chops, as heard by the observer, is the time $T_{1}$ plus the time difference for the acoustic wave to propagate from the second blade to the observer and from the first blade to the observer. The time taken 
for the acoustic wave to travel from the first blade to the observer is given by the far-field approximation of the phase radius [30] divided by the wave-speed,

$$
\tau_{0}=\frac{M_{X} X-\sigma}{c_{0} \beta^{2}} .
$$

The time taken for an acoustic wave to travel from the second blade to the observer is then obtained by replacing $Y$ in Eqn. (24) with $Y+\mathcal{C}$. The time between blade chops as heard by the observer is then given by,

$$
T=T_{1}-\frac{\mathcal{C} Y}{c_{0} \sigma} .
$$

This is the time between eddy chops as heard by the observer from the real rotor. The time between eddy chops from the image rotor is given by,

$$
T^{\#}=T_{1}-\frac{\mathcal{C} Y^{\#}}{c_{0} \sigma^{\#}} .
$$

For the first phase interference term $\left(S_{p p}^{P 1}\right)$, the time between the $0^{\text {th }}$ blade from the real rotor and the $n^{\text {th }}$ blade from the image rotor chopping an eddy is given by,

$$
T^{P 1}=T_{1}+\frac{M_{X}\left(X^{\#}-X\right)+\left(\sigma-\sigma^{\#}\right)}{c_{0} \beta^{2}}-\frac{\mathcal{C} Y^{\#}}{c_{0} \sigma^{\#}},
$$

and for the second phase interference term $\left(S_{p p}^{P 2}\right)$, the time between the $n^{\text {th }}$ blade from the real rotor and the $0^{\text {th }}$ blade from the image rotor chopping an eddy is given by,

$$
T^{P 2}=T_{1}+\frac{M_{X}\left(X-X^{\#}\right)+\left(\sigma^{\#}-\sigma\right)}{c_{0} \beta^{2}}-\frac{\mathcal{C} Y}{c_{0} \sigma} .
$$

The acoustic spectrum can now be determined for multiple blade passages by taking the Fourier transform of Eq. (एत]) [[12],

$$
S_{p p}\left(r_{0}, d_{\mathrm{wall}}, \theta, \phi, \omega\right)=\sum_{n=-\infty}^{\infty} S_{p p}^{(n)}\left(r_{0}, \theta, \phi, \omega\right) e^{\mathrm{i} n \omega T}
$$

Therefore, the PSD for a rotor with blade-to-blade correlation and with a hard- 
wall can be modelled as,

$$
\begin{aligned}
S_{p p}\left(r_{0}, d_{\text {wall }}, \theta, \phi, \omega\right)= & \sum_{j=1}^{l} \sum_{n=-\infty}^{\infty} \frac{2 \pi}{\mathcal{C}} \frac{\pi B U_{X, j} \delta r_{j}}{2}\left(k_{0} \rho_{0} b_{j}\right)^{2} \times \\
& \left\{\frac{(Y|\mathcal{L}|)^{2}}{\sigma^{4}} \Phi_{v v}\left(k_{X, j}, K_{Y, j, n}\right)+\frac{\left(Y^{\#}\left|\mathcal{L}^{\#}\right|\right)^{2}}{\left(\sigma^{\#}\right)^{4}} \Phi_{v v}\left(k_{X, j}, K_{Y, j, n}^{\#}\right)+\right. \\
& \left.\mathrm{A} \Phi_{v v}\left(k_{X, j}, K_{Y, j, n}^{P 1}\right)+\mathrm{A}^{*} \Phi_{v v}\left(k_{X, j}, K_{Y, j, n}^{P 2}\right)\right\},
\end{aligned}
$$

where quantities with the superscript \# correspond to the mirror source quantities and $k_{Y}$ is given by,

$$
K_{Y, n}=\frac{\omega T+2 \pi n}{\mathcal{C}} .
$$

In Eq. (Bत) strip theory has been used to sum the sound pressure at $l$ spanwise locations with each strip having a width $\delta r_{j}$. This strip theory is valid as long as the width of the span is much larger than the span-wise correlation length [3]. Additionally, the effect of the skewed gusts has been neglected. This implies that in the coordinate system fixed to the blade, the span-wise wavenumber, $k_{Z}$ is set to zero.

This section has included the effects of blade-to-blade correlation by considering the cross-correlation of the pressure of two blades chopping the same eddy. The next step is to determine the noise spectra of the rotating aerofoil using the extended translating aerofoil model.

\subsection{The power spectral density for the rotor}

The next step in determining the PSD for a rotating aerofoil is to apply an appropriate Doppler correction and subsequently average the PSD over all azimuthal angles in order to determine the noise spectrum of the rotating aerofoil. There are two frequencies to consider. $\omega$ is the angular frequency at the source with no relative motion between the blade and the observer and $\omega^{\prime}$ which is the Doppler shifted frequency that takes into account the motion between the source and the observer. The correction that needs to be applied is therefore 
$\omega / \omega^{\prime}$. However, this correction factor needs to be applied twice [I2, [2.9]. The reason that the correction factor needs to be applied twice is that when the PSD is averaged over the azimuth, there is no relative motion between the source and the observer. This is because the source will spend the same amount of time moving away from an observer as it does moving towards an observer. Thus, there is a Doppler correction factor for the instantaneous PSD (the PSD of the translating aerofoil) and an additional factor to account for the azimuthal averaging of the instantaneous spectrum [ [12, [29]. The PSD for the rotor is then given by,

$$
S_{p p}\left(r_{0}, \theta, \omega\right)=\frac{1}{2 \pi} \int_{0}^{2 \pi}\left(\frac{\omega}{\omega^{\prime}}\right)^{2} S_{p p}\left(r_{0}, \theta, \phi, \omega^{\prime}\right) \mathrm{d} \phi
$$

where,

$$
\frac{\omega}{\omega^{\prime}}=\left(1+\frac{\mathbf{M}_{S O} \cdot \widehat{\mathbf{S O}}}{1-\mathbf{M}_{S F} \cdot \widehat{\mathbf{S O}}}\right) .
$$

There are two Doppler factors, the Doppler shifted frequency as heard by the observer from the real rotor is $\omega^{R}$ and from the image rotor is $\omega^{I}$. These two frequencies can be computed using,

$$
\begin{aligned}
& \frac{\omega^{R}}{\omega}=1+M_{\phi} \frac{\sin \theta \sin \phi}{\sqrt{1-M_{x}^{2} \sin ^{2} \theta}}, \\
& \frac{\omega^{I}}{\omega}=1-M_{\phi} \frac{\left(r_{0}+2 d_{\text {wall }}\right) \sin \theta^{\#} \sin \phi}{r_{0} \sqrt{1-M_{x}^{2} \sin ^{2} \theta^{\#}}} .
\end{aligned}
$$

However, the phase interference terms in Eq. (प्]) contain frequencies from both the image and the real rotor. Acoustic waves that are produced by the real rotor arrive at a frequency $\omega^{R}$ while acoustic waves from the image rotor arrive at a frequency $\omega^{I}$. From Eq. (34) it is observed that the Doppler factor will be approximately 1 plus a small factor for the real rotor and 1 minus a small factor for the image rotor. Thus, the Doppler factor for the phase interference terms is approximately $1-\mathcal{O}\left(M_{\phi}^{2}\right)$. For this reason, no Doppler correction is applied 185 to the phase interference terms $\left(S_{p p}^{P 1}\right.$ and $\left.S_{p p}^{P 2}\right)$. It should be noted that the Doppler correction is still applied to the no wall $\left(S_{p p}^{N W}\right)$ and amplitude $\left(S_{p p}^{I}\right)$ terms. This approximation is valid for rotors operating at low rotational speeds. 


\subsection{Modelling the turbulence in the boundary layer}

The rotor noise model presented above determines the noise produced by a rotor near a hard-wall with blade-to-blade correlation modelled. This section presents the coupling of an anisotropic velocity spectrum with the rotor noise model. For the case of a rotor ingesting a turbulent boundary layer, it is known that the turbulent structures in the boundary layer are anisotropic [22]. Thus to accurately model the flow, the anisotropic turbulence model of Kerschen et al. [23] is used. This turbulence model is a modified Liepmann spectrum and assumes that the turbulence is axisymmetric. This assumption is valid for boundary layers, in which the turbulent eddies are cylindrical structures that are elongated in the streamwise direction [ [22]. The velocity correlation tensor is given by [2:3],

$$
\begin{aligned}
\Phi_{i j}\left(k_{x}, k_{y}, k_{z}\right)= & {\left[k^{2} \delta_{i j}-k_{i} k_{j}\right] \mathcal{F}+} \\
& {\left[\left(k^{2}-\left(k_{m} \lambda_{m}\right)^{2}\right) \delta_{i j}-k_{i} k_{j}-k^{2} \lambda_{i} \lambda_{j}+k_{m} \lambda_{m}\left(\lambda_{i} k_{j}+k_{i} \lambda_{j}\right)\right] \mathcal{G}, }
\end{aligned}
$$

where [2:3],

$$
\begin{aligned}
& k_{t}=\sqrt{k_{y}^{2}+k_{z}^{2}}, \quad z=1+l_{a}^{2} k_{x}^{2}+l_{t}^{2} k_{t}^{2}, \\
& \mathcal{F}=\frac{2 l_{a} l_{t}^{4} u_{a}^{2}}{\pi^{2} z^{3}}, \quad \mathcal{G}=\left[2 \frac{u_{t}^{2}}{u_{a}^{2}}-\frac{l_{t}^{2}}{l_{a}^{2}}-1\right] \mathcal{F},
\end{aligned}
$$

where $l_{a}$ is the axial length scale, $u_{a}$ is the axial turbulence intensity, $l_{t}$ is the transverse length scale, $u_{t}$ is the transverse turbulence intensity, and the vector $\lambda$ defines the axis of symmetry. For the case of the boundary layer the axis of symmetry is the streamwise direction and thus $\lambda=(1,0,0)$. For this model to be applicable the following criterion must be satisfied,

$$
2 \frac{u_{t}^{2}}{u_{a}^{2}}-\frac{l_{t}^{2}}{l_{a}^{2}} \geq 1 .
$$


This condition ensures that the PSD of the velocity perturbations is positive. Using Eq.(B.5), the PSD of velocity perturbations can be defined as,

$$
\begin{aligned}
\Phi_{u u}\left(k_{x}, k_{y}, k_{z}\right) & =\left[k^{2}-k_{x}^{2}\right] \mathcal{F}, \\
\Phi_{v v}\left(k_{x}, k_{y}, k_{z}\right) & =\left[k^{2}-k_{y}^{2}\right] \mathcal{F}+k_{z}^{2} \mathcal{G}, \\
\Phi_{w w}\left(k_{x}, k_{y}, k_{z}\right) & =\left[k^{2}-k_{z}^{2}\right] \mathcal{F}+k_{y}^{2} \mathcal{G} .
\end{aligned}
$$

As the axisymmetric model of Kerschen et al. [2:3] is derived in the engine-fixed reference frame, the velocity spectra in Eq. (38) need to be transformed to the blade-fixed coordinate system using the following transformation,

$$
\left(\begin{array}{c}
\tilde{\Phi}_{u u} \\
\tilde{\Phi}_{v v} \\
\tilde{\Phi}_{w w}
\end{array}\right)=\left[\begin{array}{ccc}
\cos \alpha & \sin \alpha \cos \phi & \sin \alpha \sin \phi \\
-\sin \alpha & \cos \alpha \cos \phi & \cos \alpha \sin \phi \\
0 & -\sin \phi & \cos \phi
\end{array}\right]\left(\begin{array}{c}
\Phi_{u u} \\
\Phi_{v v} \\
\Phi_{w w}
\end{array}\right),
$$

where $\left(\tilde{\Phi}_{u u}, \tilde{\Phi}_{v v}, \tilde{\Phi}_{w w}\right)$ are the velocity spectra in the blade-fixed coordinate

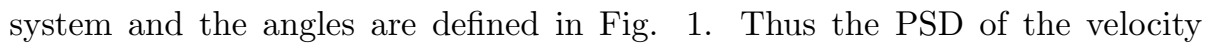
perturbations perpendicular to the blade chord is given by,

$$
\tilde{\Phi}_{v v}\left(k_{X}, k_{Y}, k_{Z}\right)=-\Phi_{u u} \sin \alpha+\Phi_{v v} \cos \alpha \cos \phi+\Phi_{w w} \cos \alpha \sin \phi .
$$

In Eq. (40), the velocity spectra on the right hand side are evaluated at the wavenumbers in the engine-fixed frame. These can be computed from the wavenumbers in the blade-fixed reference frame using the following transformation,

$$
\left(\begin{array}{l}
k_{x} \\
k_{y} \\
k_{z}
\end{array}\right)=\left[\begin{array}{ccc}
\cos \alpha & -\sin \alpha & 0 \\
\sin \alpha \cos \phi & \cos \alpha \cos \phi & -\sin \phi \\
\sin \alpha \sin \phi & \cos \alpha \sin \phi & \cos \phi
\end{array}\right]\left(\begin{array}{l}
k_{X} \\
k_{Y} \\
k_{Z}
\end{array}\right) .
$$

2.6. Numerical switch to account for the presence of the boundary layer

The formulation presented above predicts the PSD of a rotor fully immersed in a turbulent wake. The extension to Amiet's formulation must be modified to account for the fact that the blade will encounter the turbulence contained in the boundary layer only in certain parts of its rotation. To account for this, a 


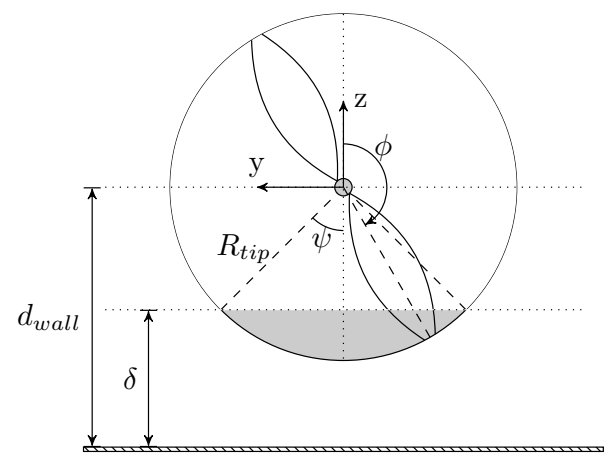

Figure 3: Schematic showing the region where a rotor blade is partially immersed in a turbulent boundary layer. $d_{\text {wall }}$ is the distance from the centre of the hub to the wall, $R$ is the distance from the hub centre to blade tip and $\delta$ is the height of the boundary layer.

numerical switch is implemented that switches the turbulence off when a rotor blade is not in the boundary layer. The geometry of the blade entering the boundary layer is shown in Fig. 3. The range of azimuthal angles that the blades will encounter turbulence in the boundary layer are given by,

$$
\pi-\psi<\phi<\pi+\psi
$$

where $\psi=\cos ^{-1}\left[\left(R_{w}-\delta\right) / R_{\text {tip }}\right], d_{\text {wall }}$ is the distance from the hub centre to the wall, $\delta$ is the height of the boundary layer, and $R_{\text {tip }}$ is the tip radius of the rotor. Additionally, when the blade interacts with the turbulent boundary layer, only a part of its span will interact with the turbulence. Thus, the sound pressure must be computed for only a few strips. The span of the blade that is immersed in the boundary layer is given by,

$$
b_{I}=R_{\mathrm{tip}}-\frac{d_{\mathrm{wall}}-\delta}{\cos (\psi-\gamma)},
$$

where $b_{I}$ is the amount of span immersed in the boundary layer and $\gamma$ is the azimuthal angle traversed by the blade in the boundary layer. Thus, Eq. (उ0) is only solved when the azimuthal angle of the blade satisfies Eq. (42) for the strips that are at a radius larger than $R_{\text {tip }}-b_{I}$.

Therefore, the noise is only predicted for a blade whose azimuthal angle satisfies Eq. (42). Additionally, the noise is only computed for strips that are 
at a radius greater than $R_{t i p}-b_{I}$.

\section{Results}

This section presents the results from the proposed rotor noise model in-

200 and the test conditions are detailed. Next, the rotor noise model is validated by comparing the noise produced by the rotor at four different advance ratios $(J)$ with available experimental data. Finally, the effect of the hard-wall on the noise produced by the rotor is determined. The effect of the hard-wall is

\subsection{Test case set-up and computational parameters}

This proposed rotor noise model requires as inputs, the geometry of the rotor, the rotor advance ratio and information that characterises the turbulence in the ingested boundary layer. The information required to characterise the and transverse directions.

The test case that was used as a benchmark was the Fundamental Case 3 (FC3) of the 2015 AIAA Fan Broadband Noise (FBN) workshop [31]. The set-up of the test case is shown in Fig. 团.

215

The FC3 benchmark case tests the rotor at 4 different advance ratios given in Tab. 四. However, at an advance ratio of $J=0.50$, a large tip vortex forms between the blade tip and the wind tunnel wall [32]. This tip vortex subsequently interacts with the rotor resulting in a large increase in the noise and large tonal peaks at the BPFs due to multiple blades passing through the same vortical structure. As the rotor noise model presented in this paper does not include the noise due to the ingestion of a tip vortex, predictions at this advance ratio were not performed.

The rotor geometry is based on a scaled Sevik rotor. The Sevik rotor has a tip diameter of $457.2 \mathrm{~mm}$ and a hub diameter of $127 \mathrm{~mm}$. The rotor has 10 


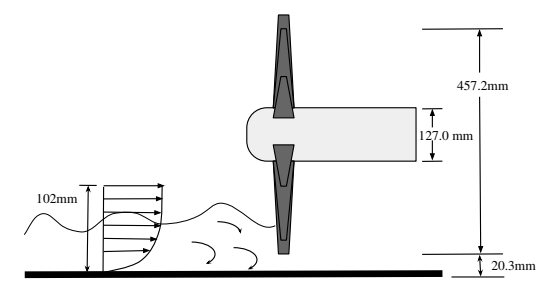

Figure 4: The setup of the test case for a rotor ingesting a turbulent boundary layer.

225 varies from $34.4^{\circ}$ at the hub to $68.8^{\circ}$ at the tip.

\begin{tabular}{ccc}
\hline Advance Ratio & Rotor speed $[\mathrm{RPM}]$ & Mean flow speed $\left[\mathrm{m} . \mathrm{s}^{-1}\right]$ \\
\hline 1.44 & 2734 & 30 \\
1.05 & 2500 & 20 \\
0.87 & 4500 & 30 \\
0.50 & 2500 & 10 \\
\hline
\end{tabular}

Table 1: The different advance ratios of the Fundamental Case 3 (FC3).

The computations for the rotor noise model are computed using 200 azimuthal integration points and 10 span-wise strips. The strips are logarithmically spaced so that the tip of the blade has a larger density of strips. The mean density and speed of sound for all computations are $1.08 \mathrm{~kg} . \mathrm{m}^{-3}$ and $350 \mathrm{~m} . \mathrm{s}^{-1}$ respectively. The microphone at which the experimental data is extracted is placed at a radius of $r_{0}=3.01 \mathrm{~m}$ and an axial angle of $\theta=127.3^{\circ}$.

\subsection{Validation of the analytical model}

In this section the extension to the rotor noise model is compared to experimental data. The predictions are made using the rotor noise model defined in 


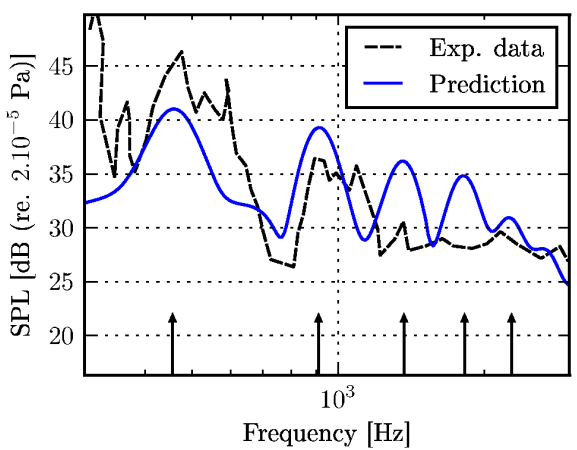

Figure 5: The PSD of the acoustic pressure for a rotor operating at the zero thrust operating condition $(J=1.44)$. The arrows represent the BPFs of the rotor. The observer is located at $r_{0}=3.01 \mathrm{~m}$ and $\theta=127.3^{\circ}$ (upstream).

Eq. (301). The Sound Pressure Level (SPL) is computed using,

$$
\mathrm{SPL}=10 \log _{10}\left(\frac{4 \pi S_{p p}\left(r_{0}, \theta, \omega\right) \Delta f}{p_{\mathrm{ref}}^{2}}\right)
$$

where $\Delta f=1 \mathrm{~Hz}$ and $p_{\text {ref }}=2 \times 10^{-5} \mathrm{~Pa}$. A factor of $2 \pi$ has been added to obtain results in $\mathrm{Hz}$ instead of rad.s $\mathrm{s}^{-1}$ and an additional factor of 2 has been added to obtain a one sided spectrum.

The characteristics of the incoming turbulence were extracted from a $4 \mathrm{D}$ time and space varying velocity correlation tensor that was obtained for an undistorted boundary layer (without the rotor present). The boundary layer height at each of the advance ratios is $102 \mathrm{~mm}$. The quantities that are required to fully characterise the anisotropic turbulence spectrum, presented in Sect. ए.5, are the turbulence intensities and integral length scales in the axial and transverse directions. The method of extracting these inputs from the experimental turbulence data provided is detailed in Appendix A. Fig. 田 shows the predicted PSD computed with the anisotropic rotor noise model with the hard-wall included. The predictions are compared to the experimental measurements of Glegg et al. [32]. There is an under-prediction at the first peak and there are peaks at the $3^{\text {rd }}$ and $4^{\text {th }}$ BPFs in the predictions that do not appear in the experimental measurements. The reasons for these discrepancies are discussed 
below.

The turbulence model of Kerschen and Gliebe [3:3] assumes that the boundary layer turbulence is axisymmetric and homogeneous. This is an approximation of the real turbulence and is a source of the discrepancies between the analytical model and the experimental data in Fig. 5. The turbulence in the boundary layer is homogeneous in the stream-wise and cross-stream directions but is inhomogeneous in the wall-normal direction. Also, as seen in Fig. A.T0, the turbulence velocities in the transverse directions $(v, w)$ are not the same for $R / R_{\text {tip }}>0.75$. Thus the turbulence is not universally axisymmetric. Fig. A.10 also shows that the assumptions in the anisotropic turbulence model of Kerschen and Gliebe [33] are increasingly valid as one moves further away from the wall.

As stated previously, the turbulence in the boundary layer is homogeneous in the stream-wise and cross-stream directions and thus length scales can be obtained directly in these directions. However, the transverse integral length scale must be chosen empirically due to the inhomogeneity in the wall normal direction. The value for the transverse transverse integral length scale used in this study is $0.03 \mathrm{~m}$. This is close to the value of the transverse length scale at the rotor hub as shown in Fig. A.T0. Also, the transverse length scale does not vary significantly at various wall normal positions as shown in Fig. 270 A.10(c). Thus, the transverse length scale used seems a reasonable choice for this study. All other parameters for this validation study are taken directly from the experimental measurements.

Fig. 6 shows the PSD computed using the anisotropic rotor noise model with the hard-wall included with the empirical transverse length scale of $l_{t}=0.03$ m. The predictions are made for three different advance ratios. The predicted results are compared with the experimental data of Glegg et al. [32].

Fig. 6 shows the general agreement between the predictions and the experimental measurements at the three advance ratios. The peak amplitudes at the first BPF are close to the measured values for the $J=1.44$ and $J=1.05$ advance ratios, while the amplitude of the first BPF is over-predicted at $J=0.87$. The 
amplitude of the second BPF is slightly over predicted at all the advance ratios. Additionally, the amplitude of the spectra after the $2^{\text {nd }} \mathrm{BPF}$ is over-predicted for the $J=1.44$ advance ratio. At the $J=1.44$ and $J=1.05$ advance ratios there is a drop off at high frequencies in the predictions compared to the experiments. The high frequency drop off could potentially be due to additional sources in the experiments that are not included in the prediction model. It could also be due to the high frequency slope of the turbulence model of Kerschen and Gliebe [33] not matching the boundary layer turbulence decay. For the $J=1.05$ advance ratio, the $2^{\text {nd }}$ and $3^{\text {rd }} \mathrm{BPF}$ are not at the same location as the measured spectrum. The BPFs of the measured spectra for the $J=1.05$ advance ratio are not at the expected $\mathrm{BPF}$ of the rotor. A possible explanation of this could potentially be due to the vortical structures being ingested by the rotor being inclined $[\underline{9}]$. In spite of the differences discussed above, the agreement is within the expected accuracy of a semi-empirical analytical model.

The individual terms in Eq. (Bत) can be analysed to see their contribution to the total noise spectra. Fig. $\square$ shows the amplitude of the individual cross spectral terms of Eq. (BII) for a rotor operating at the zero thrust $(J=1.44)$ operating condition. It is observed that the amplitude of the sum of the phase interference terms between the rotor and the image rotor $\left(S_{p p}^{P 1}\right.$ and $\left.S_{p p}^{P 2}\right)$ contribute to the spectrum between the blade passing frequencies and subtract from the spectrum at the blade passing frequencies. The increase in noise in the spectra at frequencies less than the $1^{\text {st }} \mathrm{BPF}$ is due to these phase interference terms. The amplitude term due to the image rotor $\left(S_{p p}^{I}\right)$ has the BPFs in the same location as the no-wall term (Amiet's [ए2] original formulation) and thus is only increasing the amplitude of the spectrum at this observer angle. The over-prediction at frequencies above the second BPF is caused by the fall-off of the no-wall term not decaying after the $2^{\text {nd }} \mathrm{BPF}$. The amplitude term due to the image rotor $\left(S_{p p}^{I}\right)$ also broadens the BPF peaks slightly as the peak is not exactly at the BPF of the no-wall contribution. Equations [5.5 and [26 show that the time between eddy chops and subsequently the peak frequency are dependent on the distance to the observer in the reference frame fixed to the blade. 


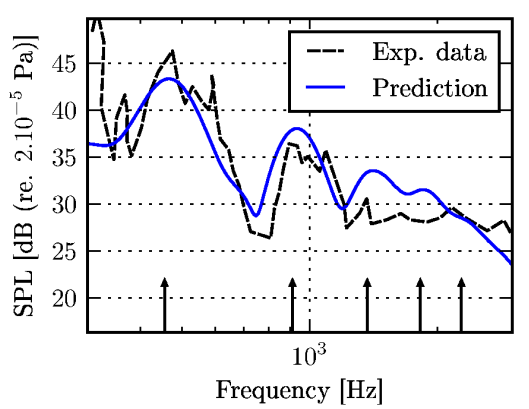

(a)

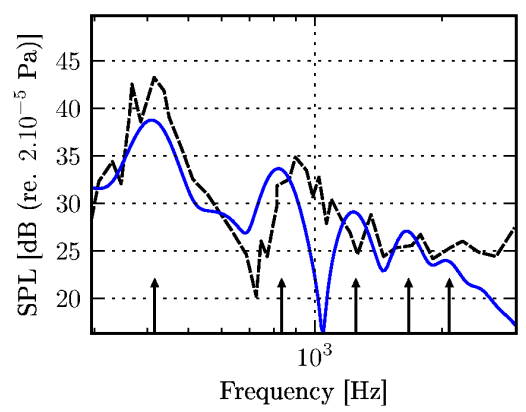

(b)

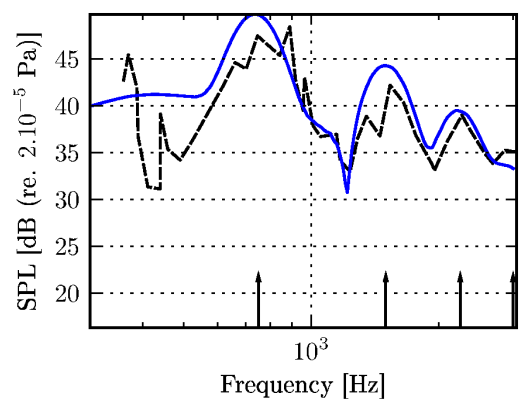

(c)

Figure 6: The PSD of the acoustic pressure for a rotor operating at 3 different operating conditions. The arrows represent the BPFs of the rotor. The observer is located at $r_{0}=3.01$ m and $\theta=127.3^{\circ}$ (upstream). (a) $J=1.44$; (b) $J=1.05$; (c) $J=0.87$.

These times will therefore be different for the real and image observer.

The bandwidth of the haystacks at the BPF are known to scale with $U_{x} / l_{a}$ [23]. As the free stream velocity for the $J=1.44$ and the $J=0.87$ advance ratios is the same and the free stream velocity for the $J=1.05$ is similar to the other two advance ratios, it is expected that the bandwidth of the haystacks should be similar at all three advance ratios as is observed in Fig. [6.

Figs. 8(a), 8(d), and 8(g) show the SPL of the rotor varying with observer angle and frequency at three different advance ratios. These contour maps show the dipole like directivity of the rotor with a cusp at the $90^{\circ}$ observer angle. It is also observed that the SPL increases as the advance ratio increases. This 


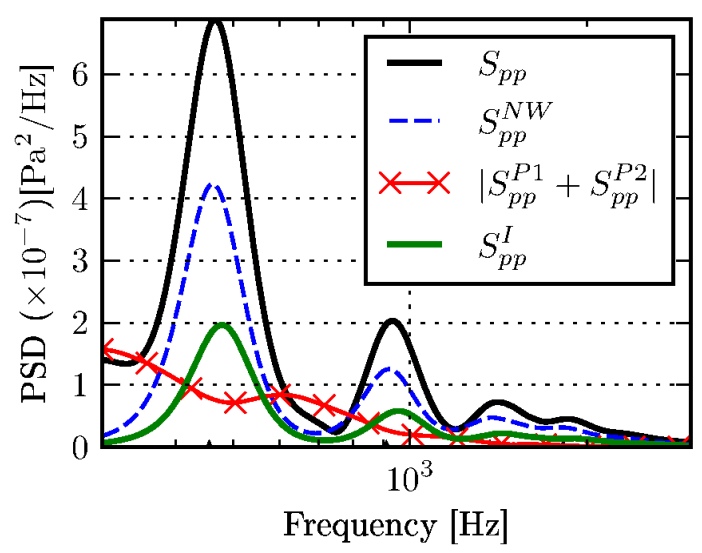

Figure 7: The individual terms of Eq. (四). The rotor is operating at the zero thrust advance ratio of $J=1.44$ and the observer is at $\left(r_{0}, \theta\right)=\left(3.01 \mathrm{~m}, 127.3^{\circ}\right)$. The arrows indicate the BPFs of the rotor.

is due to the velocity relative to the blade chord $\left(U_{X}\right)$ increasing as the rotor RPM is increased.

The OverAll Sound Pressure Level (OASPL) is computed between $300 \mathrm{~Hz}$ and $3 \mathrm{kHz}$ as follows,

$$
\mathrm{OASPL}=10 \log _{10}\left(\frac{4 \pi \int_{f=300 \mathrm{~Hz}}^{f=3000 \mathrm{~Hz}} S_{p p}\left(r_{0}, \theta, \omega\right) \mathrm{d} f}{p_{\text {ref }}^{2}}\right) .
$$

The measured and predicted OASPLs are computed for the first three advance 325 ratios and these are tabulated in Tab. 20. It is observed that the OASPL is under-predicted at the first two advance ratios and over-predicted at the third advance ratio.

\begin{tabular}{cccc}
\hline Advance ratio & Measured OASPL $(\mathrm{dB})$ & Predicted OASPL $(\mathrm{dB})$ & DOASPL (measured-predicted) $(\mathrm{dB})$ \\
\hline 1.44 & 69.42 & 68.35 & +1.06 \\
1.05 & 65.03 & 62.54 & +2.49 \\
0.87 & 74.14 & 75.71 & -1.58 \\
\hline
\end{tabular}

Table 2: The measured and predicted OASPLs at three different advance ratios.

It should be noted that the rotor noise model is compared to published 
experimental data that was available at one microphone location. The predicted OASPLs and the location of the BPFs are again reproduced to a level of accuracy that is expected for a semi-empirical analytical method. The next section will investigate the effect of the installation of the rotor by examining the noise produced by a rotor with and without a hard-wall.

\subsection{Installation effects}

In this section the effect of the hard-wall on the noise is examined. This effect is examined at three different advance ratios. To quantify the effect that the wall has on the rotor noise, predictions for the rotor noise are computed with and without the wall.

Fig. $\mathbb{8}$ shows the PSD of the sound varying with observer angle and frequency for three different advance ratios. The rotor noise is computed with the wall (Figs. 8(a), 8(d), and 8(g)) and without the wall (Figs. 8(b), 8(e), and 8(h)) Figs. 8(c), 8(f), and 8(i) show the difference in the sound produced by the rotor with and without a hard-wall.

Fig. $\varangle$ shows that the main effect of the hard-wall is to modulate the amplitude of the spectra. The presence of the hard-wall does not significantly alter the directivity of the rotor or the locations of the BPFs. The differences between with and without a wall (Figs. 8(c), 8(f), and 8(i)) show that the hard-wall has the largest contribution around the cusp of the BPFs. Additionally, there is a large increase in the low-frequency sound in the spectra before the $1^{\text {st }} \mathrm{BPF}$. As has been discussed previously, this increase is due to the interference terms and is also shown in the experimental measurements. The increase in SPL is more than $6 \mathrm{~dB}$ at certain frequencies and observer angles. Two reasons are hypothesised for this. First, at locations of destructive interference, the sound power with a hard-wall tends to 0 due to the destructive interference. This could give a difference of greater than $6 \mathrm{~dB}$ between the cases with and without the hard-wall. Second, the increase of greater than $6 \mathrm{~dB}$ could be due to directivity effects caused by the numerical switch introduced in Sect. 2.6. Also the image rotor is rotating in the opposite direction to the real rotor. This results in 


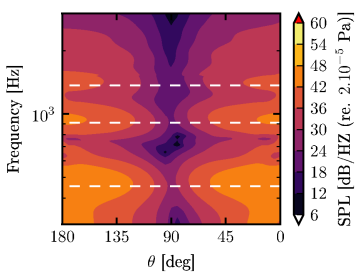

(a)

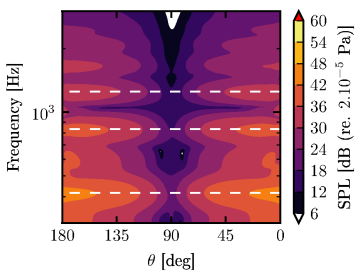

(d)

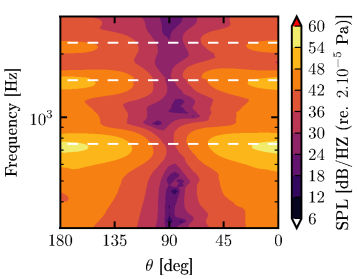

(g)

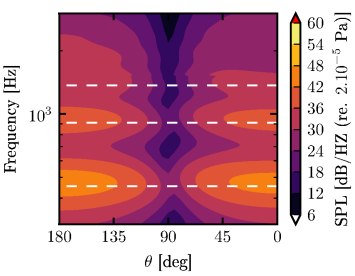

(b)

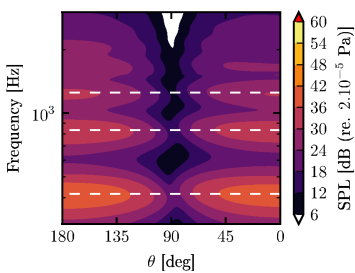

(e)

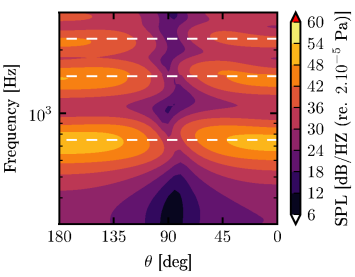

(h)

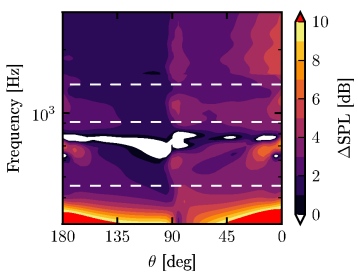

(c)

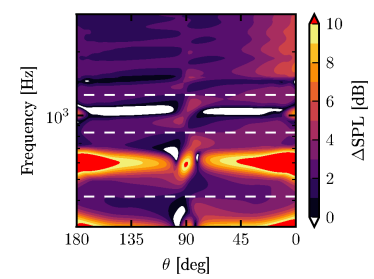

(f)

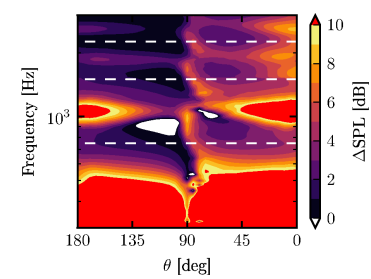

(i)

Figure 8: Contours of the PSD of the acoustic pressure for a rotor varying with observer angle $\left(0^{\circ}\right.$ points downstream $)$ and frequency. A transverse length scale of $l_{t}=0.03 \mathrm{~m}$ is used. The observer is at $r_{0}=3.01 \mathrm{~m}$ and the hard-wall is located at $d_{\text {wall }}=0.2486 \mathrm{~m}$. The dashed lines represent the BPFs of the rotor. (a) PSD with a hard-wall at $J=1.44$; (b) PSD without a wall at $J=1.44$; (c) Difference in PSD at $J=1.44$; (d) PSD with a hard-wall at $J=1.05$; (e) PSD without a wall at $J=1.05$; (f) Difference in PSD at $J=1.05$; (g) PSD with a hard-wall at $J=0.87$; (h) PSD without a wall at $J=0.87$; (i) Difference in PSD at $J=0.87$.

opposing directivity functions that could lead to the greater than $6 \mathrm{~dB}$ increase in noise that is observed.

Fig. 9 shows directivity plots of the OASPL for the three different advance ratios and the difference between them. The rotor shows a dipole like directivity with a slightly louder OASPL at the downstream observers. It is observed that 


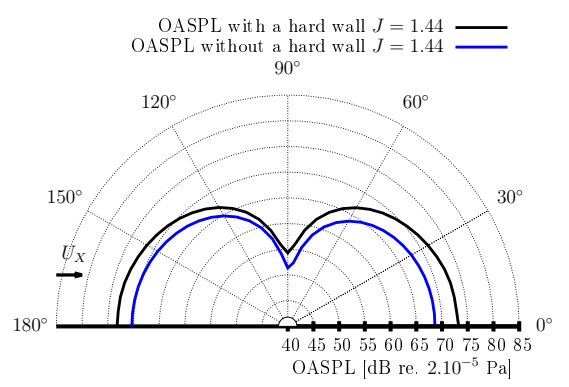

(a)

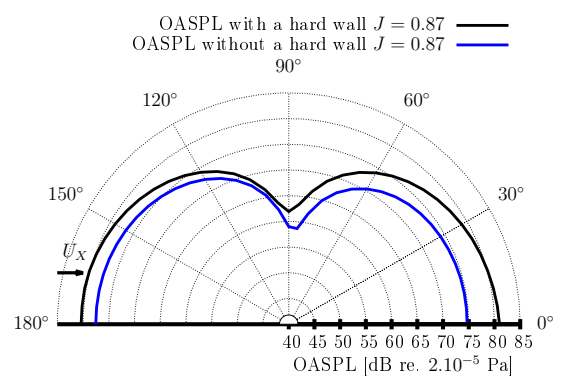

(c)

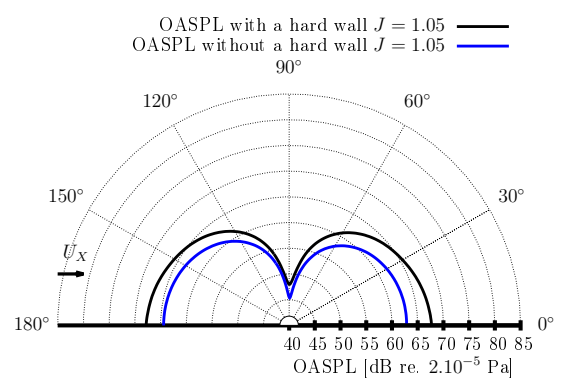

(b)

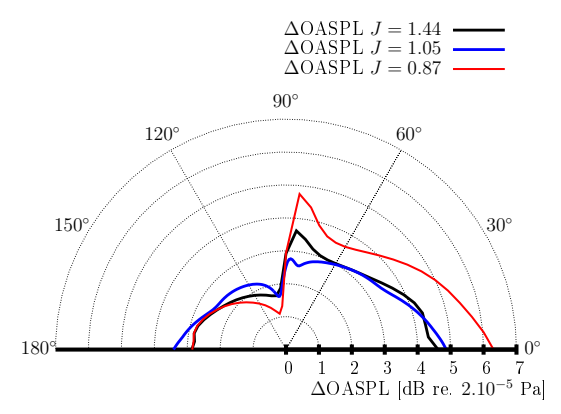

(d)

Figure 9: OASPL directivity of the PSD of the acoustic pressure for a rotor operating at 3 different advance ratios. (a)OASPL $\mathrm{J}=1.44$; (b) OASPL $\mathrm{J}=1.05$; (c) OASPL $\mathrm{J}=0.87$; (d) $\triangle$ OASPL.

the wall increases the OASPL by approximately $3 \mathrm{~dB}$ for the upstream observers and by $5-6 \mathrm{~dB}$ for the downstream observers. The higher rotor RPM is shown to increase the effect of the wall a little more for the downstream observers as compared to the lower RPMs.

The results in this section have shown that the reflecting plane can have a significant impact on the noise produced by the rotor. It is observed that the largest noise increase is at the low frequencies before the first BPF and in the cusp between the $1^{\text {st }}$ and $2^{\text {nd }} \mathrm{BPF}$. The OASPL directivities show that the rotor produces more noise at the downstream observer with the addition of a hard wall and hence the downstream directivity is more significantly affected by the 
wall. Increasing the rotor RPM increases the noise amplification effect of the hard-wall slightly.

\section{Conclusions}

This paper has investigated the noise produced by an open rotor ingesting a boundary layer in proximity to an infinite flat wall. This noise source is likely to be a dominant noise source in open rotors that are installed close to an aircraft fuselage.

This paper has extended Amiet's simplified rotor noise model to include this noise source. To accurately model this noise source, the blade-to-blade correlation, the effect of the reflecting surface and the anisotropy of the boundary layer have all been modelled. In order to include these effects three extensions have been made to Amiet's simplified rotor model. The method of images has been used to model the hard-wall. The anisotropic turbulence model of Kerschen and Gliebe [2:3] has been used to model the anisotropy in the boundary layer and finally a numerical switch has been introduced in order to compute the noise only when the rotor blade is passing through the boundary layer.

The developed rotor noise model is computationally cheap (a few minutes on a personal computer) and requires as inputs, a description of the turbulence that can be obtained from a time resolved numerical simulation for example. While, the transverse length scale must be chosen empirically, the model is fast enough to enable the computation of rotor noise at various length scales. As the turbulence in the boundary layer is not homogeneous in the wall-normal direction, an empirical value for the transverse length scale must be chosen. The addition of a hard-wall in the rotor noise model ensures that the predicted spectrum levels take into account acoustic reflections from an infinite flat-plate. The addition of an anisotropic spectrum ensures that the energy in the spectrum is distributed correctly and this combined with a blade-to-blade correlation model reproduces the characteristic haystacks that are present in the spectrum of this noise source. The relative simplicity of the developed rotor noise model also 
ensures that the physics of this noise mechanism can be understood.

The rotor noise model has been validated using experimental data at three advance ratios from the FC3 case of the FBN workshop. The proposed model predicts the acoustic spectrum and the location of the BPFs to a degree of accuracy that is acceptable for a semi-empirical analytical model.

The effect of the hard-wall on the noise produced by the rotor is investigated at three different advance ratios. At all advance ratios it is found that the hard-wall has a significant effect on the installed noise of the open rotor at an observer angle of $90^{\circ}$. This is most prominent at low frequencies. This increase is approximately $6 \mathrm{~dB}$. It is also observed that the hard-wall affects the downstream directivity more significantly than the upstream directivity. Changing the operating condition of the rotor does not significantly alter the 415 effect of the hard-wall on the noise spectrum. 


\section{Appendix A. Extraction of turbulence data from the test case to in- put into the rotor noise model}

The predictions of the rotor noise model are computed for the Fundamental Case 3 (FC3) of the 2015 AIAA Fan Broadband Noise (FBN) workshop

The turbulence intensities in the longitudinal and transverse directions at each wall normal location are computed by obtaining the velocity correlation at zero time and zero span-wise separation,

$$
\left(\frac{u_{i}^{\prime}(z)}{U}\right)^{2}=R_{i i}(0,0, z, z) .
$$

To obtain the integral length scales in the longitudinal and transverse directions, normalised velocity correlation functions were computed from the experimental data. The integral length scales were determined by integrating these velocity correlations. The results obtained were validated by comparing the experimentally determined velocity correlations with analytical velocity corre- 
lations. Two longitudinal velocity correlations $f_{x}$ and $f_{y}$ can be defined for the Liepmann spectrum as [34, 3.5],

$$
\begin{aligned}
& f_{x}(r)=e^{-|s| / L_{11}^{(1)}}, \\
& f_{y}(r)=e^{-|s| / L_{22}^{(2)}},
\end{aligned}
$$

where $s$ is the separation between the two points at which the correlation is considered, $L_{11}^{(1)}$ is the integral length scale computed from the correlation function of the $u$ velocity separated in the $x$ direction, and $L_{22}^{(2)}$ is the integral length scale computed from the correlation of the $v$ velocity separated in the $y$ direction. As these velocity correlations take the integral length scale computed from experimental data as an input, comparing these velocity correlations with experimental data will indicate the accuracy of the determined length scales. A similar method cannot be used to extract the integral length scales in the wall-normal direction as the turbulence is not homogeneous in this direction.

The computed normalised velocity correlations in the streamwise $(x)$ and the cross-stream $(y)$ directions are shown in Fig. 10(a) and Fig. 10(b) respectively. It can be seen that the velocity correlations extracted from the experimental data compare well with those computed using Eq. A.2.

The computed integral length scales are shown in Fig. 10(c) as a function of 450 the radial location from the hub centre. The ratio of the integral length scales near the rotor hub is approximately 6 and approximately 2 near the rotor tip. Fig. 10(d) shows the variation of the turbulence intensity as a function of radial location from the rotor hub centre. As expected, the streamwise turbulence intensities are considerably larger than in the other directions. Additionally, the turbulence intensities decrease monotonically away from the wall. To facilitate the use of this data in the rotor noise model, least square fits were computed for the integral length scales as,

$$
\begin{aligned}
& L_{11}^{(1)}=-0.4681 z+0.0947, \\
& L_{22}^{(2)}=0.0958 z+0.0116,
\end{aligned}
$$




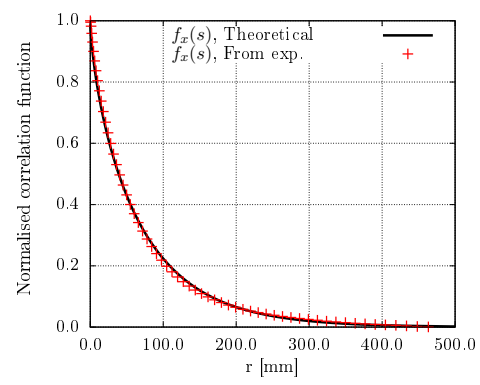

(a)

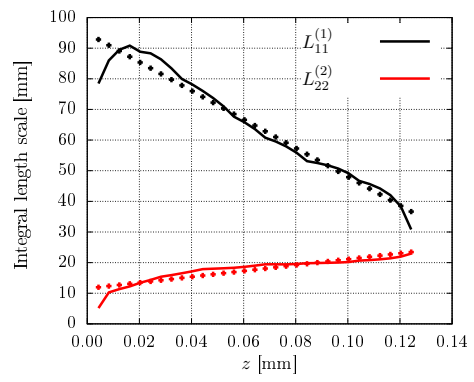

(c)

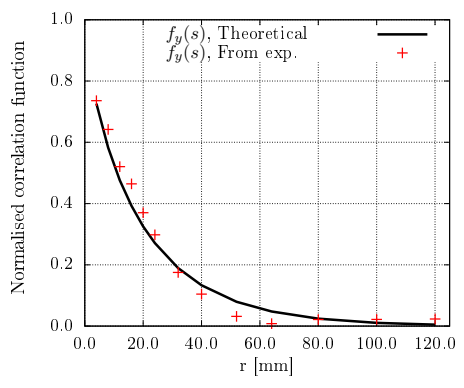

(b)

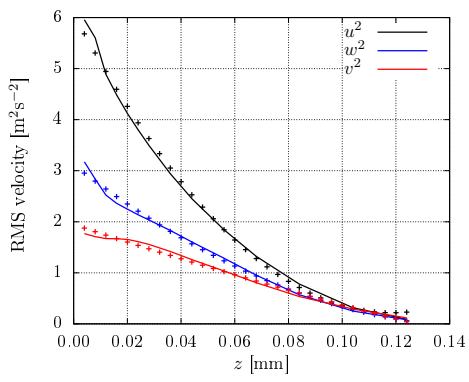

(d)

Figure A.10: The integral length scales, RMS velocities, and normalised correlation functions. The points show the least square fits for each of the variables computed using Eqs.

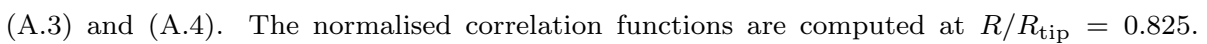
(a)Streamwise correlation function; (b)Cross-stream correlation function; (c)Integral length scales; (d)Turbulence velocities.

and the turbulence intensities as,

$$
\begin{aligned}
& u^{2}=417.2 z^{2}-98.83 z+6.070, \\
& w^{2}=131.7 z^{2}-40.96 z+3.115, \\
& v^{2}=16.23 z^{2}-17.35 z+1.944
\end{aligned}
$$

where $z$ is the distance from the wall in meters. In the rotor noise model presented, $L_{11}^{(1)}=l_{a}, L_{22}^{(2)}=l_{t}, u=u_{a}$, and $\sqrt{\left(v^{2}+w^{2}\right) / 2}=u_{t}$. Additionally, all the turbulence data is scaled by the free-stream velocity.

The values for the axial and transverse turbulence intensities and integral length scales $\left(u_{a}, l_{a}, u_{t}, l_{t}\right)$ computed from Eqs. (A.3) and (A.4) are computed 
at every strip. These are then used in Eq. (Bत) to determine the noise radiated at that strip.

\section{References}

[1] A. Parry, M. Kingan, B. Tester, Relative importance of open rotor tone and broadband noise sources, in: 17th AIAA/CEAS Aeroacoustics Conference, AIAA 2011-2763, Portland, USA, 2011. doi: $10.2514 / 6.2011-2763$. URL http://arc.aiaa.org/doi/10.2514/6.2011-2763

[2] Flightpath 2050 Europe's Vision for Aviation, Tech. rep., High Level Group on Aviation Research, Directorate-General for Mobility and Transport, Directorate-General for Research and Innovation (2011). doi:10.2777/50266. URL https://publications.europa.eu/en/publication-detail/-/ publication/296a9bd7-fef9-4ae8-82c4-a21ff48be673

[3] V. P. Blandeau, P. F. Joseph, Broadband Noise Due to Rotor-Wake/Rotor Interaction in Contra-Rotating Open Rotors, AIAA Journal 48 (11) (2010) 2674-2686. doi:10.2514/1.J050566.

URL http://arc.aiaa.org/doi/abs/10.2514/1.J050566

[4] T. Nodé-Langlois, F. Wlassow, V. Languille, Y. Colin, B. Caruelle, J. R. Gill, X. Chen, X. Zhang, A. B. Parry, Prediction of Contra-Rotating Open Rotor broadband noise in isolated and installed contigurations, in: 20th AIAA/CEAS Aeroacoustics Conference, AIAA 2014-2610, Atlanta, Georgia, 2014. doi:10.2514/6.2014-2610.

URL http://arc.aiaa.org/doi/abs/10.2514/6.2014-2610

[5] P. Block, Counter-rotating propeller noise directivity and trends, in: AIAA 10th Aeroacoustics Conference, AIAA 86-1927, Seattle, USA, 1986. doi:10.2514/6.1986-1929. 
[6] J. Ricouard, E. Julliard, M. Omais, V. Regnier, A. Parry, S. Baralon, Installation Effects on Contra-Rotating Open Rotor Noise, in: 16th AIAA/CEAS Aeroacoustics Conference, AIAA 2010-3795, Stockholm, Sweeden, 2010. doi:10.2514/6.2010-3795.

URL http://arc.aiaa.org/doi/pdf/10.2514/6.2010-3795

495

[7] W. N. Alexander, W. J. Devenport, D. Wisda, M. A. Morton, S. A. Glegg, Sound Radiated from a Rotor and Its Relation to Rotating Frame Measurements of Ingested Turbulence, in: 20th AIAA/CEAS Aeroacoustics Conference, AIAA 2014-2746, Atlanta, Georgia, 2014. doi:10.2514/6.2014-2746. URL http://arc.aiaa.org/doi/abs/10.2514/6.2014-2746

[8] D. B. Stephens, S. C. Morris, Sound Generation by a Rotor Interacting with a Casing 'Turbulent Boundary Layer, AIAA Journal 47 (11) (2009) 2698-2708. doi:10.2514/1.43271. URL http://arc.aiaa.org/doi/10.2514/1.43271

[9] D. Wisda, W. N. Alexander, W. J. Devenport, S. A. Glegg, Boundary Layer Ingestion Noise and Turbulence Scale Analysis at High and Low Advance Ratios, in: 20th AIAA/CEAS Aeroacoustics Conference, AIAA 2014-2608, Atlanta, Georgia, 2014. doi:10.2514/6.2014-2608. URL http://arc.aiaa.org/doi/abs/10.2514/6.2014-2608

[10] S. A. Glegg, W. Devenport, N. Alexander, Broadband rotor noise predictions using a time domain approach, Journal of Sound and Vibration 335 (2015) 115-124. doi:10.1016/j.jsv.2014.09.007. URL http://linkinghub.elsevier.com/retrieve/pii/S0022460X14007202

[11] M. J. Czech, R. H. Thomas, Open Rotor Aeroacoustic Installation Effects for Conventional and Unconventional Airframes, in: 9th AIAA/CEAS Aeroacoustics Conference, AIAA 2013-2185, Berlin, Germany, 2013. doi:10.2514/6.2013-2185. 
[12] R. Amiet, Noise Produced by Turbulent Flow Into a Rotor: Theory Manual for Noise Calculation, Tech. Rep. June 1989, United Technologies Research Center (1989).

[13] R. Amiet, Noise Produced by turbulent flow into a propeller or helicopter rotor, in: 3rd AIAA Aeroacoustics Conference, AIAA-P-76-560, Paolo Alto, California, 1976.

[14] S. Moreau, M. Roger, Competing Broadband Noise Mechanisms in Low-Speed Axial Fans, AIAA Journal 45 (1) (2007) 48-57. doi: $10.2514 / 1.14583$. URL http://arc.aiaa.org/doi/10.2514/1.14583

[15] A. Pagano, M. Barbarino, D. Casalino, L. Federico, Tonal and Broadband Noise Calculations for Aeroacoustic Optimization of a Pusher Propeller, Journal of Aircraft 47 (3) (2010) 835-848. doi:10.2514/1.45315. URL http://arc.aiaa.org/doi/10.2514/1.45315

[16] R. W. Paterson, R. K. Amiet, Noise of a model helicopter rotor due to ingestion of turbulence, Tech. rep., NASA Langley Research Center (1979).

[17] R. H. Schlinker, R. K. Amiet, Helicopter Rotor Trailing Edge Noise, Tech. rep., NASA Langley Research Center (1981).

[18] S. Glegg, S. Baxter, A. Glendinning, The prediction of broadband noise from wind turbines, Journal of Sound and Vibration 118 (2) (1987) 217-239. doi:10.1016/0022-460X(87)90522-0.

URL http://linkinghub.elsevier.com/retrieve/pii/0022460X87905220

[19] A. D. Pierce, Acoustics. An Introduction to its Physical Principles and Applications, Acoustical Society of America, 1981.

[20] K. Kucukcoskun, J. Christophe, C. Schram, M. Tournour, A Semi-Analytical Approach on the Turbulence Interaction Noise of A 
Low-Speed Axial Fan Including Broadband Scattering, in: 17th

AIAA/CEAS Aeroacoustics Conference, AIAA 2011-2714, Portland, Oregon, 2011. doi:10.2514/6.2011-2714.

URL http://arc.aiaa.org/doi/abs/10.2514/6.2011-2714

[21] K. Kucukcoskun, Prediction of tree and scattered acoustic tields of low-speed tans, Ph.D. thesis, Ecole Centrale De Lyon (2012).

URL https://tel.archives-ouvertes.fr/tel-00758274

[22] M. Gad-el Hak, P. R. Bandyopadhyay, Reynolds Number Effects in Wall-Bounded Turbulent F'lows, Applied Mechanics Reviews 47 (8) (1994) 307-365. doi:10.1115/1.3111083.

URL http://appliedmechanicsreviews.asmedigitalcollection. asme.org/article.aspx?articleid=1395357

[23] E. J. Kerschen, P. R. Gliebe, Noise Caused by the Interaction of a Rotor with Anisotropic Turbulence, AIAA Journal 19 (6) (1981) 717-723. doi:10.2514/3.50995. URL http://arc.aiaa.org/doi/abs/10.2514/3.50995

[24] S. B. Pope, Turbulent Flows, Cambridge University Press, 2001.

[25] I. Marusic, B. J. McKeon, P. A. Monkewitz, H. M. Nagib, A. J. Smits, K. R. Sreenivasan, Wall-bounded turbulent flows at high Reynolds numbers: Recent advances and key issues, Physics of Fluids 22 (6) (2010) 1-24. doi:10.1063/1.3453711.

[26] S. A. Glegg, The Response of a Swept Blade Row to a Three-Dimensional Gust, Journal of Sound and Vibration 227 (1) (1999) 29-64. doi:10.1006/jsvi.1999.2327. URL http: //wWW.sciencedirect.com/science/article/pii/S0022460X99923271

[27] H. Posson, S. Moreau, M. Roger, Broadband noise prediction of fan outlet guide vane using a cascade response function, Journal of Sound and 
Vibration 330 (25) (2011) 6153-6183. doi:10.1016/j.jsv.2011.07.040.

URL http://dx.doi.org/10.1016/j.jsv.2011.07.040

[28] R. Amiet, Acoustic Radiation From an Airfoil in a Turbulent Stream, Journal of Sound and Vibration 41 (4) (1975) 407-420.

doi:10.1016/S0022-460X(75)80105-2.

URL http:

//wWw.sciencedirect.com/science/article/pii/S0022460X75801052

[29] S. Sinayoko, M. Kingan, A. Agarwal, Trailing edge noise theory for rotating blades in uniform How, Proceedings of the Royal Society A: Mathematical, Physical and Engineering Sciences 469 (2157) (2013) 1-21. arXıV:1304.80/2, doi:10.1098/rspa.2013.0065.

URL http://rspa.royalsocietypublishing.org/cgi/doi/10.1098/ rspa.2013.0065

[30] I. Garrick, C. Watkins, A Theoretical Study of the Effect of Forward Speed on the Free-Space Sound Pressure Field Around Propellers, Tech. Rep. October, National Advisory Committee for Aeronautics Langley Aeronautical Laboratory (1953).

[31] Fan Broadband Noise Prediction Panel Session at 21st AIAA/CEAS Aeroacoustics Conference.

URL http://web1.oai.org/FBNWorkshop.nsf/Indexaccessedon01/06/2015

[32] S. A. Glegg, J. Grant, H. Murray, W. J. Devenport, W. N. Alexander, Sound Radiation from a Rotor Operating at High Thrust Near a Wall, in: 22nd AIAA/CEAS Aeroacoustics Conference, AIAA 2016-2995, Lyon, France, 2016. doi:10.2514/6.2016-2995. URL http://arc.aiaa.org/doi/10.2514/6.2016-2995

[33] E. Kerschen, P. Gliebe, Fan noise caused by the ingestion of anisotropic turbulence - A model based on axisymmetric turbulence theory, in: 6th 
Aeroacoustics Conference, AIAA-80-1021, Hartford, Connecticut, 1980.

doi:doi:10.2514/6.1980-1021.

[34] D. K. Wilson, Turbulence models and the synthesis of random fields for acoustic wave propagation calculations, Tech. rep., Army Research Laboratory (1998).

[35] H. W. Liepmann, J. Laufer, K. Liepmann, On the spectrum of isotropic turbulence, Tech. rep., NACA (1951). 